\title{
Kommunikation als kreativer Prozess: Im Dialog mit dem Kunden
}

\author{
Riklef Rambow
}

Die Kommunikation mit dem Bauherren [1] ist ein wesentlicher Teil des Entwurfsprozesses in der Architektur. Auch wenn sich wohl jeder Architekt schon einmal gewünscht hat, es möge anders sein, so gilt doch: Die Wünsche und Erwartungen des Bauherren bilden die Voraussetzung jeder konkreten Architektur. Ohne die Schnittstelle zum Bauherren bliebe die Architektur auf dem Skizzenblock gefangen. Durch Kommunikation erfolgt die aktive Gestaltung dieser Schnittstelle. Die Wünsche, Vorstellungen und Erwartungen von Bauherren sind nicht starr und unveränderlich. Sie entwickeln sich dynamisch im gesamten Verlauf des Planungs- und Bauprozesses, und die Art dieser Entwicklung wird im Wesentlichen durch die Kommunikation des Architekten bestimmt. Deshalb ist es wichtig, dass diese zielgerichtet, transparent, verlässlich und überzeugend erfolgt.

\section{Experten-Laien-Kommunikation}

Psychologisch betrachtet, handelt es sich bei der Kommunikation zwischen Architekt und Bauherren in aller Regel um einen Fall von Experten-LaienKommunikation. Dabei übernimmt der Architekt die Position des Experten, und der Bauherr ist in der Position des Laien. Die Kommunikation ist dadurch im Kern asymmetrisch: Der Architekt als Experte für Architektur weiß mehr über dieses Thema: Er kann vieles, was der Laie nicht kann, er denkt anders darüber, er nimmt Architektur anders wahr, und er kommt zu anderen Bewertungen. Sein Denken und Sprechen ist durch Fachkonzepte geprägt, die er sich in einem lang währenden Prozess angeeignet hat.

Der Bauherr als Laie kennt diese fachlich geprägte Perspektive auf Architektur nicht. Er kann sie auch nicht wirklich abschätzen, dafür fehlen inm das erforderliche Wissen und die nötigen Kompetenzen. Stattdessen ist er auf recht vage Vermutungen darüber angewiesen, wie ein Architekt die Welt und - vor allem - die Architektur sieht. Und natürlich auf die Kommunikation des Experten. Der Architekt muss sich verständlich machen. Er darf seine fachliche Perspektive nicht als die einzig richtige Sichtweise voraussetzen, sondern muss sein Wissen und seine Einschätzungen so vermitteln, dass sie auch auf der Grundlage der ganz anders gearteten, lücken- und manchmal auch fehlerhaften Sichtweise eines Laien nachvollziehbar erscheinen. Mit anderen Worten: Die grundlegende
Asymmetrie der Experten-Laien-Kommunikation hat zur Folge, dass die Hauptverantwortung für das Gelingen der Kommunikation beim Architekten liegt. Von inm kann erwartet werden, dass er in der Lage ist, die Perspektive des Laien abzuschätzen, aber umgekehrt gilt das nur sehr eingeschränkt. Der Architekt muss sich auf den Bauherren einstellen, um dessen Perspektive zu verstehen, und sie im kontinuierlichen Dialog gezielt weiterzuentwickeln.

\section{Kommunikation ist ein Prozess}

Die Voraussetzungen von Kommunikation unterscheiden sich deutlich in den verschiedenen Phasen eines Kommunikationsprozesses. Während der ersten Begegnung mit dem Bauherren ist der Architekt bei der Abschätzung von dessen Perspektive auf stereotypes Wissen und auf direkte Rückmeldungen angewiesen. Im weiteren Verlauf der Arbeitsbeziehung gewinnt er ein immer genaueres Bild von den Wünschen und Gewohnheiten, den Vorstellungen vom Leben und Wohnen, den ästhetischen Ideen und wichtigen Bezugsobjekten des Bauherren. Der Architekt entwickelt in dieser Phase ein mentales »Partnermodell «, auf das er seine Kommunikation abstimmen kann. Aus dem anonymen Exemplar der Spezies »Bauherr « wird für inn eine konkrete Person mit einem bestimmten Profil. Auf dieser Grundlage entwickelt er, ohne darüber bewusst nachdenken zu müssen, im Gespräch 
spontane Vermutungen darüber, was der Bauherr erwartet und wie er auf Äußerungen des Architekten reagieren wird. Das ist ein großer Vorteil für die Kommunikation, birgt aber auch Gefahren. Ein solches "Partnermodell « kann grob und fehlerhaft sein und es kann schnell »erstarren «. Nur allzu oft haben wir schon nach kurzer Bekanntschaft das Gefühl, unser Gegenüber nun sicher einschätzen zu können, wir haben bestimmte Gepflogenheiten und Redeweisen wiederholt wahrgenommen und sie scheinen mit unseren allgemeinen Erwartungen gut zusammen zu passen. Das dynamische "Partnermodell « ist aus Bequemlichkeit zum Klischee erstarrt, es ist statisch geworden. Das Problem dabei ist, dass solche Klischees in der Kommunikation wie ein Aufmerksamkeitsfilter wirken, der nur Informationen durchlässt, die mit dem Klischee übereinstimmen, während er inkongruente Informationen ausblendet.

Um den Prozesscharakter von Kommunikation zu erhalten, muss der Architekt dieser Erstarrung gezielt und bewusst entgegen arbeiten.

Er sollte in jeder Phase der Beziehung bereit sein, seine Vermutungen an der Realität zu prüfen, indem er dem Bauherren Gelegenheit gibt, seine Vorstellungen zu bestimmten Aspekten des Projekts zu äußern. Die Chance zur Veränderung von Auffassungen besteht nur, wenn die Situation und die sich daraus ergebenden Möglichkeiten immer wieder vorurteilsfrei zur Diskussion gestellt werden.

\section{Erwartungen des Bauherren}

Erwartungen bilden sich auf der Grundlage von allgemeinem Wissen und konkreten Vorerfahrungen. Daher haben viele private Bauherren nur recht vage Vorstellungen davon, was Architekten tun und was sie können. Nicht selten ist der Erstkontakt im Rahmen eines Projekts für den Bauherren auch das erste Mal, dass er überhaupt mit einem Architekten zu tun hat. Zu diesem Zeitpunkt hat er sich wahrscheinlich durch private Empfehlung, im Internet oder durch die Lek-

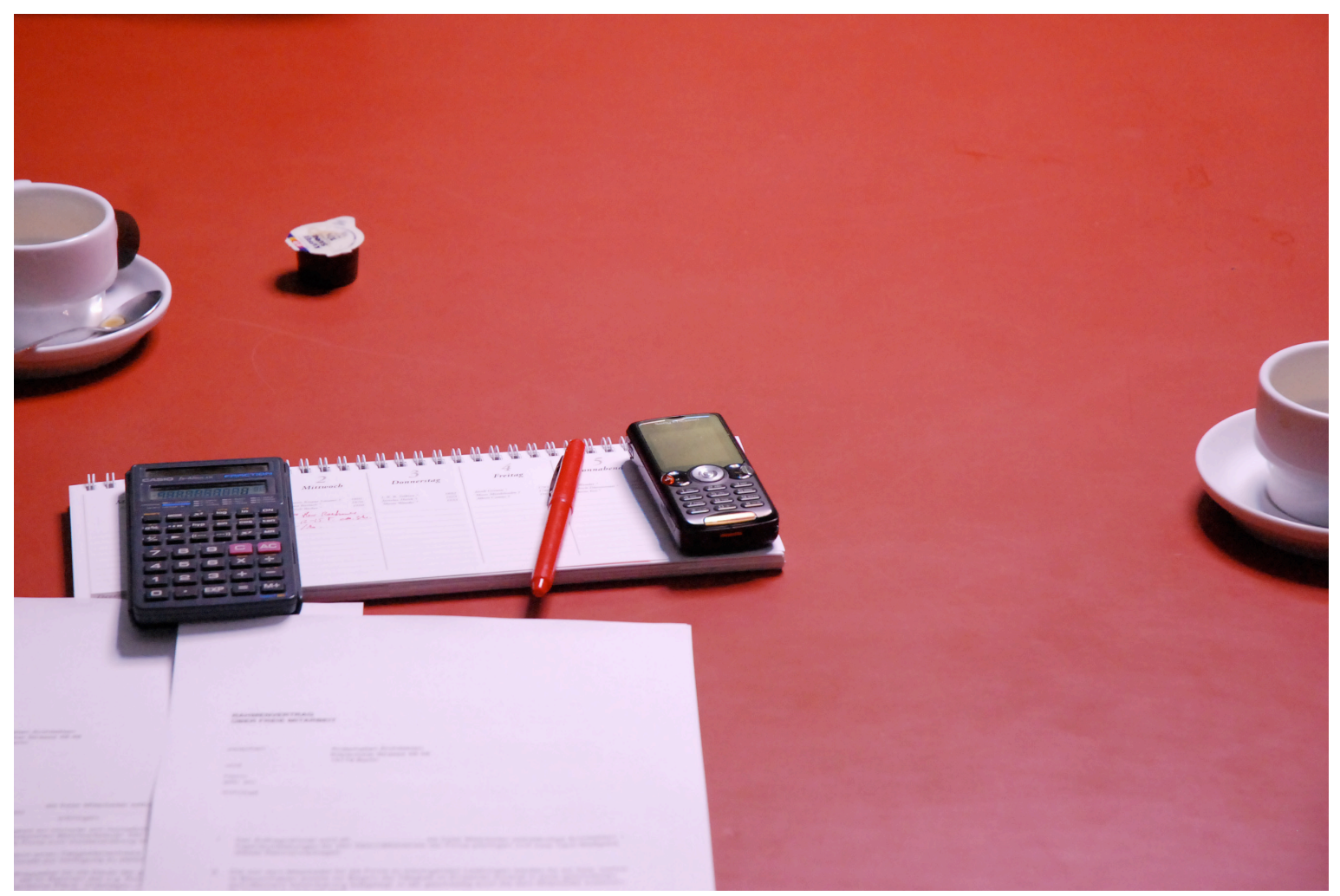


türe von Zeitschriften bereits erste Informationen verschafft. Er dürfte kaum gänzlich unvorbereitet sein, denn sonst hätte er vermutlich gar nicht erst den Weg zum Architekten gewählt, sondern sich, wie die Mehrzahl der angehenden Bauherren, an einen Bauträger oder Fertighausanbieter gewandt.

Die Tatsache, dass er den Weg zum Architekten gewählt hat, impliziert für den Bauherren zwei voneinander getrennte Entscheidungen: Erstens diejenige, überhaupt einen Architekten zu beauftragen, und zweitens die Entscheidung, genau diesen bestimmten Architekten aufzusuchen. Beide Entscheidungen sind zum Zeitpunkt des Erstkontakts sehr wahrscheinlich noch mit Unsicherheiten und Befürchtungen behaftet. Der Architekt muss während der ersten Gespräche darauf eingehen. Dabei tut er gut daran, die beiden Aspekte sorgfältig getrennt zu behandeln. Es geht also zunächst darum, deutlich zu machen, welche Leistungen das Berufsbild Architekt generell beinhaltet und welche nicht, welche Regeln im Umgang zwischen Architekt und Bauherren gelten und wie die Rechte und Pflichten verteilt sind.

Im zweiten Schritt kommt die individuelle Qualifikation ins Spiel. Erst jetzt muss der Architekt zeigen, wie er selbst die Freiräume ausfüllt, die das Berufsbild inm eröffnet. Die Trennung der beiden Aspekte ist für den Aufbau einer vertrauensvollen Arbeitsbeziehung wichtig, weil sie dem Bauherren ermöglicht, zwischen allgemeinen Rahmenbedingungen und individuellen Entscheidungen des Architekten zu unterscheiden. Er kann so die Freiheitsgrade im Handeln des Architekten realistisch einschätzen - eine wichtige Voraussetzung, um die Leistung des Architekten fair beurteilen zu können.

Die allgemeine Aufklärung über das Berufsbild und das Leistungsspektrum von Architekten muss die zum Teil diffusen Erwartungen klären, die der Bauherr bereits mitbringt. Das heißt, sie muss auch den besonders machtvollen Klischees entgegenwirken, die von Nicht-Architekten mit dem Berufsbild verbunden werden. Untersuchungen zeigen, dass Bauherren vom Architekten vor allem Kosten- und Terminsicherheit, Zuverlässigkeit und eine umfassende Koordination der verschiedenen am Bau beteiligten Parteien erwarten. Kreativität und Originalität spielen dagegen eine untergeordnete Rolle bzw. werden oftmals einfach vorausgesetzt. Die schwerwiegendste Befürchtung besteht dementsprechend - vereinfacht ausgedrückt - darin, an eine eigensinnige KünstlerDiva zu geraten, die Kosten und Termine den eigenen kreativen Interessen unterordnet. Architekten, so das Klischee, gewichten funktionale und wirtschaftliche Erwägungen niedrig, ästhetische Ziele dagegen hoch. Sie orientieren sich an den Werten ihrer Fachkollegen und interessieren sich nicht für die Urteile von Laien. Diesen Vorstellungen muss der Architekt bei den ersten Kontakten mit dem Bauherren entgegenwirken. Entscheidend hierfür ist eine sachliche, respektvolle Haltung dem Bauherren gegenüber, die deutlich signalisiert, dass es zunächst einmal ausschließlich um dessen Motivationen, Wünsche und Vorstellungen geht. Gefragt ist hierbei eine Haltung des aktiven Zuhörens. Der Architekt stellt offene Fragen, vermeidet eigene Wertungen, und registriert die Antworten seines Gegenübers sorgfältig. Empfehlenswert für diese Gespräche ist die Entwicklung einer schriftlichen Protokolltechnik, die - für den Bauherren sichtbar - alle wichtigen Punkte festhält. Kurze Rückfragen dienen der Klärung eventueller Missverständnisse. Gelegentliche Paraphrasierungen, also das Wiederholen der Äußerungen des Bauherren mit eigenen Worten, dienen dazu, eine gemeinsame Sprache zu finden. Das Ziel dieser »diagnostischen « Kommunikation besteht darin, möglichst viele Informationen über die Absichten und Kenntnisse des Bauherrn zu sammeln, und zugleich Interesse an sowie Respekt gegenüber seiner Person zu vermitteln. Der Impuls, sich selbst als kreative und kompetente Persönlichkeit zu präsentieren, sollte demgegenüber zunächst zurückstehen. Für den privaten Bauherren ist die Entscheidung zu bauen fast immer von erheblicher Tragweite. In vielen Fällen handelt es sich um eines der wichtigsten und folgenschwersten Projekte seines Lebens. Bezogen auf die finanziellen Kosten ist es in der Regel die größte Investition, die der Bauherr überhaupt je tätigen wird. Einem solchen Schritt gehen komplexe persönliche Entscheidungsprozesse voraus, an denen neben dem Bauherren selbst auch noch weitere Familienmitglieder beteiligt sind. Diese Prozesse können sich über einen beträchtlichen Zeitraum erstrecken. Der Erstkontakt mit dem Architekten markiert also aus der Perspektive des Bauherren keineswegs den Anfang 
des Projekts, sondern viel eher den Abschluss einer persönlichen, oftmals von einer komplexen sozialen Dynamik geprägten Vorplanungsphase. Mit anderen Worten: Es gibt auf Seiten des Bauherren zu diesem Zeitpunkt bereits ein ganzes Bündel an Vorstellungen über das Projekt, die nur zum Teil verhandelbar sind. Nicht alle diese Vorstellungen wird der Bauherr bereits bei den ersten Kontakten mit dem Architekten auch äußern, sei es, weil sie inm selbst gar nicht wirklich bewusst sind oder weil er glaubt, sie hätten mit dem Projekt gar nichts zu tun, sei es, weil er sie aus strategischen Überlegungen noch zurückhält. Der Architekt muss diese Zurückhaltung akzeptieren. Er sollte aber dennoch von Anfang an große Aufmerksamkeit auf die unausgesprochenen persönlichen und sozialen Hintergründe und Motivationen verwenden. Mit wachsendem Vertrauen in der Beziehung zwischen Bauherren und Architekt können diese Rahmenbedingungen dann sorgfältiger exploriert werden.

\section{Die Präsentation der eigenen Person und des Büros}

Natürlich möchte auch der Bauherr wissen, mit wem er es zu tun hat, bevor er sich für den Architekten entscheidet. Bei der Präsentation der eigenen Person und des eigenen Büros sollte der Architekt im Auge behalten, welche Aspekte aus Sicht des Bauherren besonders wichtig sind, und welche Sorgen und Befürchtungen es zu entkräften gilt. Im Zentrum sollte deshalb zunächst die Arbeitsweise des Büros stehen und weniger das bisherige Werk. Dabei meint Arbeitsweise weniger das, was gemeinhin als "Philosophie" eines Architekturbüros, z.B. auf dessen Internetseite, formuliert wird, als vielmehr konkrete Prozeduren im Umgang mit einer Aufgabenstellung. Noch einmal sei auf die existenzielle Bedeutung von Kosten- und Terminsicherheit für den Bauherren hingewiesen. Wie diese im Büro gewährleistet werden, wie die interne Aufgabenteilung funktioniert, wer der Ansprechpartner des Bauherren sein wird, - all das sind Punkte, die unaufdringlich zur Sprache kommen sollten. Natürlich sind auch Referenzprojekte wichtig, allerdings weniger unter dem Aspekt, die eigene künstlerische "Handschrift « zu illustrieren, als vielmehr, um die oben genannten prozeduralen Aspekte zu belegen. Der Bauherr möchte sehen, dass der Architekt mit der Art von Problem, die sein Projekt beinhaltet, kompetent und souverän umgehen kann. Für eine solche Urteilsbildung sind Informationen wie Bausumme, Bauzeit und Auftraggeber von Bedeutung. Sie dürfen schon bei der Projektdarstellung im Internet nicht fehlen. Desgleichen sollten gute Arbeitsbeziehungen mit Spezialisten, Baufirmen und Handwerkern Erwähnung finden, die als erprobtes Netzwerk Sicherheit für die Realisierung des Projekts bieten. Wichtig bei dieser ersten Büropräsentation ist das ständige Bewusstsein des Architekten, dass viele der üblichen Prozesse und Strukturen dem Bauherren unbekannt oder nur in Umrissen verständlich sind.

\section{Vertrauen}

Eine der wesentlichen Voraussetzungen und zugleich ein Ziel von Kommunikation ist der Aufbau gegenseitigen Vertrauens. Dies ist umso wichtiger, wenn es sich, wie im Fall von Architekt und Bauherren, um eine Beziehung handelt, die sich über einen langen Zeitraum erstreckt und bei der einiges auf dem Spiel steht. Vertrauen in den Architekten entsteht, wenn sein kommunikatives Verhalten als transparent, verlässlich und kompetent wahrgenommen wird. Transparenz beruht auf Verständlichkeit und Nachvollziehbarkeit. Nachvollziehbarkeit bedeutet, dass der Architekt dem Bauherren zu keinem Zeitpunkt wichtige Informationen bewusst vorenthält oder sie einfach voraussetzt. Der Bauherr will stets einschätzen können, warum der Architekt bestimmte Entscheidungen trifft und welche Konsequenzen daraus erwachsen. Da diese Entscheidungen häufig auf der Grundlage von Vorkenntnissen getroffen werden, die bis dahin nur dem Architekten zugänglich und präsent sind, muss dieser von sich aus diese Zugänglichkeit durch Erklärungen herstellen.

Darüber hinaus sollte der Architekt alles tun, um als kompetent wahrgenommen zu werden. Die Zuschreibung von Kompetenz hängt von verschiedenen Faktoren ab. Sie beginnt häufig schon vor dem Erstkontakt durch die Empfehlungen von Freunden oder Bekannten. Auch die Darstellung der eigenen Arbeit, z.B auf der eigenen Website, kann den Eindruck von Kompetenz verstärken, nämlich dann, wenn der Bauherr dort Referenzprojekte findet, die er als relevant für seine eigene Aufgabenstellung empfindet. 


\section{Präsentation von Projekten und Ideen}

Eine Kommunikationssituation von oft entscheidender Bedeutung ist die Projektpräsentation. Im Kern geht es immer darum, dass innerhalb relativ kurzer Zeit durch einen konzentrierten, medienunterstützten Vortrag ein architektonisches Konzept so darzustellen ist, dass es von den Zuhörern als überzeugende Problemlösung beurteilt wird. Idealerweise hat die Wirkung der Präsentation etwas Zwingendes: danach sollten die Zuhörer das Bedürfnis verspüren, das Projekt zu realisieren.

Um in kurzer Zeit eine solche Wirkung zu erzielen, muss die Präsentation präzise und zielgruppengenau geplant und umgesetzt werden. Häufig kommt die Chance einer solchen Präsentation, einmal vertan, nicht so schnell wieder. Aber auch bei Zwischenpräsentationen in späteren Phasen eines Projekts, also nach erfolgter Beauftragung, ist ihre Bedeutung für die Beziehung zwischen Architekt und Bauherren stets hoch und rechtfertigt allemal eine sorgfältige Vorbereitung.

Der Kern jeder Präsentation ist eine überzeugende, logisch aufgebaute Argumentationsstruktur. Es reicht nicht, so wie es häufig im Bereich der Architektur üblich ist, ein Konzept oder ein Projekt einfach zu zeigen; stattdessen muss jede wichtige Entwurfsentscheidung schlüssig begründet werden. Es reicht auch nicht, vor allem auf die Macht der Bilder zu vertrauen. Stattdessen müssen Worte und Bilder konsequent aufeinander bezogen sein und beide stets

\section{info}

\section{Überzeugungsarbeit im Dialog}

Planungen und Ideen erfolgreich präsentieren

- Hinweise für Architekten und Planer für eine

Präsentation im kleinen Kreis:

(von Riklef Rambow und Frank Peter Jäger)

Vor jeder Präsentation sollten Sie deren Ziel klar bestimmt haben.

- Jede Präsentation hat eine sachliche und eine emotionale Ebene. Wenn es gelingt, die Sympathie des Gesprächspartners für Ihre Person zu wecken, wird er auch ein offenes Ohr für Ihre Sachargumente haben.

- Vieles spricht dafür, Präsentationen zu zweit durchzuführen, - vor allem, wenn auch die Kundenseite mit zwei oder mehr Personen vertreten ist. Während der eine spricht, kann sich der andere auf die nonverbalen Reaktionen der Gesprächspartner konzentrieren. Abgesehen von den rhetorischen Vorteilen des Duos bieten zwei Personen zudem ein doppeltes Identifikationspotenzial.

- Weil es darauf ankommt, den Gesprächspartner für Ihre Sache zu gewinnen, muss man inm eine Planung nicht bis ins letzte Detail er- klären. Haben Sie Mut zur Lücke und bringen Sie zunächst die wesentlichen Gesichtspunkte zur Sprache - pointiert und ohne komplizierte Fachbegriffe.

- Dabei helfen starke Bilder. Gefragt sind anschauliche Visualisierungen - allerdings nur wenige, wirklich gute - denn es ist fatal, jemanden, der sich gerade für Ihre Gedanken öffnet, mit einer Flut von Material zu ersticken.

- Erklären Sie Ihre Bilder, belegen Sie Ihre Worte. Erst in ihrem genau abgestimmten Zusammenspiel entfalten Bild und Wort ihre optimale Wirkung.

- Verbinden Sie jedes Element Ihrer Präsentation (z.B. eines Entwurfs) mit schlüssigen Argumenten, sodass jede Ihrer planerischen Entscheidungen nachvollziehbar und folgerichtig erscheint.

- Ist ein Präsentationstermin bei einem potenziellen Bauherren auf 45 Minuten angesetzt, bietet sich folgende zeitliche Gliederung an: Drei Minuten für die Begrüßung und das Bestimmen der Gesprächsziele; fünf Minuten für die Vorstellung Ihres Büros; 15 Minuten für 
im Dienst der Argumentationsstruktur stehen. Jede bildliche Darstellung, ob Plan, Perspektive, Modellfoto oder Simulation, bildet ein visuelles Argument, das für die zuhörenden Laien oft nicht aus sich allein heraus verständlich ist, sondern durch gezielte Erläuterungen ergänzt werden muss.

Am Anfang jeder Präsentation steht die Problemdarstellung oder vielmehr Probleminterpretation. Die Analyse programmatischer, städtebaulicher, rechtlicher, ökonomischer und weiterer Rahmenbedingungen muss so auf wenige, präzise skizzierte Parameter reduziert werden, dass sich alle Aspekte der folgenden Konzeptdarstellung eindeutig darauf beziehen lassen. Nur was in der Probleminterpretation als relevant definiert wird, braucht bei der Beschreibung des Lösungsansatzes erwähnt zu werden. Am Ende der Präsentation muss der Kreis explizit oder implizit geschlossen werden, sodass jeder Zuhörer die Überzeugung gewinnt, dass Probleminterpretation und Lösungsansatz geschmeidig ineinander greifen wie Schloss und Schlüssel.

Bildliche Darstellungen spielen dabei eine zentrale Rolle. Ein häufiger Fehler besteht darin, zu viele Abbildungen einzusetzen. Die Absicht ist dabei wohl, dass sich aus vielen kurzen Eindrücken wie in einer Art Kaleidoskop ein umfassender, positiv getönter Gesamteindruck ergeben möge. Die Abbildungen werden dabei nicht im Dienste der Argumentation eingesetzt, sondern als offene Assoziationsangebote. Diese Rechnung kann zwar in Einzelfällen durchaus aufge- die Präsentation Ihres Projektkonzepts; die verbleibende Zeit ist Rückfragen und dem gemeinsamen Gespräch vorbehalten.

- Ihr eigener Wortanteil im Gespräch sollte 50 \% nicht überschreiten. Gelegentliche Rückfragen und Paraphrasierungen (Wiederholungen der Aussagen Ihres Gesprächspartners in eigenen Worten) stellen sicher, dass Sie dessen Anliegen wirklich verstanden haben.

- Ermuntern Sie Gesprächspartner zu Rückfragen und eigenen Anmerkungen; eine gekonnte Präsentation ist kein Monolog, sondern eine Diskussionsplattform: Sie gibt Ihnen Aufschluss, wie Ihre Ideen beim Gesprächspartner ankommen.

- Notieren Sie sich die Fragen und Einwände des Gesprächspartners, denn sie bilden die Grundlage für die spätere Überarbeitung Ihres Entwurfskonzeptes. Zugleich sieht Ihr Bauherr seine Anliegen in Ihre Überlegungen integriert.

- Power-Point-Charts sind ein gutes Medium für Präsentationen vor größeren Gruppen. Sitzen Ihnen nur zwei oder drei Gesprächspartner gegenüber, ist eine gedruckte, auf dem Tisch ausgebreitete Präsentation die bessere Wahl, denn sie ist "greifbarer « und wirkt persönlicher.

- Entscheiden Sie schon bei der Vorbereitung einer solchen Tischvorlage, welche Teile davon Sie dem Bauherren danach überlassen. Gestalten Sie diese so, dass sie in sich klar und unmissverständlich sind, denn der Bauherr wird sie nutzen, um Dritten über das Projekt zu berichten.

- Als visuelle Entsprechung zu den Paraphrasierungen kann der Architekt mit einem Bleistift die Ideen des Bauherren in seinem Plan skizzieren. Auf der Sachebene werden dessen Vorstellungen dadurch zum beiderseitigen Verständnis konkretisiert, auf der emotionalen Ebene sieht der Bauherr seine Belange aktiv ins Gespräch einbezogen.

- Jede Stehgreif-Skizze hat Charme, weil sich in ihr für den Laien die Kreativität des Architekten mit den eigenen Ideen verbindet, - und das entspricht genau der Rollenerwartung an den Architekten. 
hen, führt aber auf lange Sicht zu Problemen. Sie setzt nicht auf Überzeugung, sondern auf Überrumpelung. Bildliche Darstellungen in der Architektur sind fast immer komplex und mehrdeutig. Ihre Dekodierung braucht Zeit und Unterstützung. Die Zeit bei Präsentationen ist eng beschränkt, daher sollte auch die Anzahl der visuellen Darstellungen gering sein. Jede Darstellung erfüllt eine klare argumentative Aufgabe, also muss man ihr auch genug Zeit lassen, um auf den Betrachter zu wirken.

Während eine Abbildung gezeigt wird, sollte sich der Vortragende konsequent auf sie beziehen. Er lenkt die Aufmerksamkeit der Zuhörenden, indem er erläutert, was man auf der Abbildung sieht, inwiefern bestimmte Aspekte abstrahiert sind, welche besonderen Qualitäten des Entwurfs in der Darstellung anschaulich werden. Da dem Zuhörer das Vorwissen des Vortragenden fehlt, sind Zeigebewegungen, eindeutige Beschriftungen, Zooming etc. besonders wichtig. Ohne solche Hilfestellungen - die natürlich nicht oberlehrerhaft daherkommen dürfen, sondern als eine reibungslose, unaufdringliche Begleitung durch die Komplexität des eigenen Entwurfs - ist die Gefahr von Missverständnissen erheblich.

Die schwierigste Aufgabe bei der Planung einer Präsentation ist die Beschränkung auf das Wesentliche. Für die meisten Architekten wäre es überhaupt kein Problem, aus dem Stegreif stundenlang über das eigene Konzept zu sprechen, immerhin ist es das Resultat wochenlanger intensiver Beschäftigung. Aber gerade das ist nicht möglich, stattdessen ist auch hier - wie so oft - weniger mehr. Die erfolgreiche Vorbereitung einer Präsentation erfordert die Bereitschaft, das eigene Konzept noch einmal mit neuen Augen zu sehen. Es gilt, durch die verschlungenen Wege seiner Entstehung nachträglich einen roten Faden zu legen. Dabei sollte konsequent die Perspektive des Bauherren berücksichtigt werden: Für inn geht es nicht um ein architektonisches Konzept, sondern um ein Gebäude, das bestimmte Anforderungen

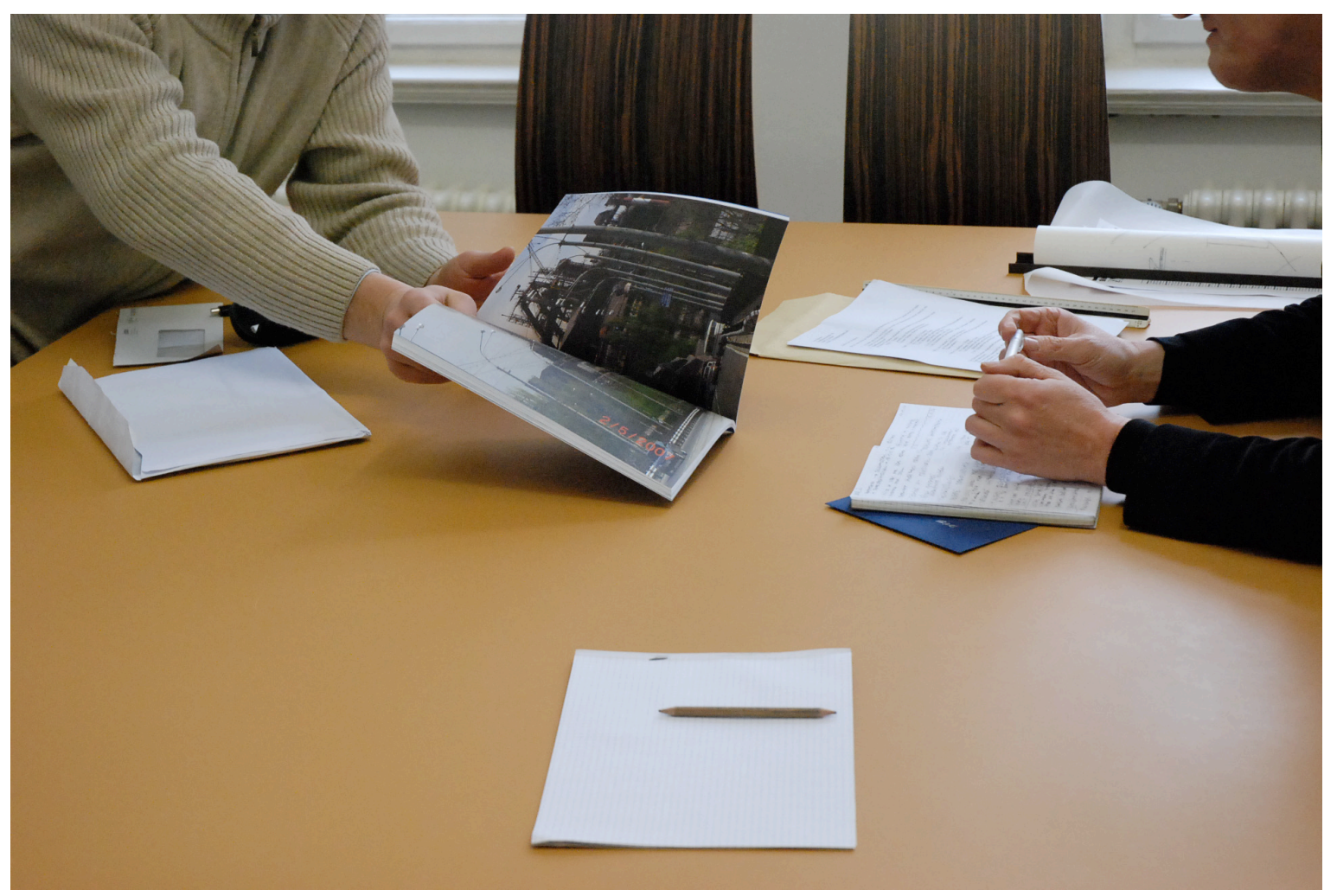


möglichst gut erfüllt. Es geht um Kosten für Energie und Instandhaltung, also um Nachhaltigkeit im umfassenden Sinne. Es geht nicht um abstrakte Funktionen, sondern um konkrete Handlungs- und Erlebnismöglichkeiten, um das alltägliche Wohnen, das Miteinander mit der Familie, um die mögliche Anpassung an zukünftige Veränderungen. Es geht möglicherweise um Repräsentation im öffentlichen Raum, die mit dem Selbstbild des Bauherren übereinstimmt und diesem eine neue, angemessene Gestalt gibt.

\section{Kommunikation verändern}

Das sprachliche Kommunikationsverhalten in realen Dialogsituationen ist in hohem Maße routinisiert und automatisiert. Bestimmte Angewohnheiten wie eingeschliffene Redewendungen, Floskeln, Akzentuierungen und Satzmelodien, aber auch nonverbale Kommunikationsformen wie gestische und mimische Ausdrucksweisen, sind über Jahrzehnte erlernt worden und deshalb nur mit hohem Aufwand und viel Übung veränderbar. Zudem kommt eine weitere Schwierigkeit hinzu: In den wenigsten Situationen bekommen wir eine direkte Rückmeldung über den Erfolg oder Misserfolg unserer Kommunikation. Gerade ein solches Feedback ist aber die Grundvoraussetzung für gezielte Verbesserungen. Möchte man sein professionelles Kommunikationsverhalten verbessern, sind deshalb die folgenden drei Faustregeln nützlich:

- Die Energie sollte weniger in die Kommunikation selbst, als vor allem in deren gründliche Vorbereitung investiert werden. Denn bei der Gestaltung von Hilfsmitteln wie Broschüren, Websites, Präsentationsfolien und der zugehörigen Texte kann man sich die Zeit nehmen, systematischer über die Bedürfnisse und den Wissensstand des Bauherren nachzudenken, die Ziele der Kommunikation klar zu definieren und die ausgewählten Medien dementsprechend zu gestalten.

- Zweitens sollte, wann immer möglich, die eigene Wahrnehmung durch das Urteil von Kollegen und Mitarbeitern überprüft und ggf. korrigiert werden. Das betrifft die Präsentationsmaterialien ebenso wie den wichtigen Besprechungstermin, bei dem ein Partner protokollieren und seine Beobach- tungen in einem Nachgespräch schildern kann. Voraussetzung für eine ehrliche Rückmeldung, die immer auch kritische Äußerungen beinhalten kann, ist ein hinreichend offenes und vertrauensvolles Verhältnis zwischen den Beteiligten.

- Drittens sollte die Möglichkeit nicht verschenkt werden, die Äußerungen des Bauherren selbst als Informationsquelle über das eigene Kommunikationsverhalten zu nutzen. Sie erschließt man über die bereits erwähnten Techniken des Fragens und aktiven Zuhörens. In allen Phasen des Kommunikationsprozesses sollte der Architekt in der Lage sein, die gewohnte Expertenrolle vorübergehend zu verlassen, und dem Bauherren die Gelegenheit geben, sein aktuelles Problemverständnis zu artikulieren. Denn gerade die "naiven Theorien « des Bauherren bieten dem Architekten die Chance, seine eigenen kommunikativen Strategien und Instrumente zu schärfen und zielgenau zu optimieren.

\section{Fazit}

Erfolgreiche professionelle Kommunikation ist keine Fähigkeit, die man in einigen wenigen Regeln zusammenfassen und über Nacht erlernen kann. Sie ist eine lebenslange Herausforderung, die immer wieder aufs Neue die Überprüfung und kreative Weiterentwicklung scheinbar altbewährter Strategien verlangt. Wenn sie aber gelingt, dann ermöglicht sie, durch echten Dialog eine glückliche Synthese aus den Bedürfnissen des Bauherren und den Möglichkeiten der Architektur zu finden.

\section{Anmerkung:}

[1] Der Einfachheit halber ist in diesem Text durchgängig von Architekt und Bauherr die Rede. Damit sind sowohl männliche wie weibliche Personen angesprochen. Die Ausführungen beziehen sich in erster Linie auf private Bauherren. Eine Diskussion von Unterschieden zwischen privaten und gewerblichen bzw. institutionellen Bauherren kann in diesem Rahmen nicht geleistet werden. Vieles, was im Text beschrieben wird, dürfte aber mit geringen Modifikationen auch für letztere gelten. 